\title{
Erratum to: Multiplex detection of food allergens and gluten
}

\author{
Chung Y. Cho ${ }^{1} \cdot$ William Nowatzke $^{2} \cdot{\text { Kerry } \text { Oliver }^{2} \text { • Eric A. E. Garber }}^{1}$
}

Received: 22 June 2016 / Accepted: 22 June 2016 / Published online: 26 July 2016

(C) Springer-Verlag Berlin Heidelberg 2016

\section{Erratum to: Anal Bioanal Chem}

DOI 10.1007/s00216-015-8645-y

In the original version of this article unfortunately, Table 2 contained some errors in the placement of the values. Below, please find the corrected version; in the electronic version, readers can easily enlarge it to meet their reading needs.

The online version of the original article can be found at http:// dx.doi.org/10.1007/s00216-015-8645-y.

\footnotetext{
Eric A. E. Garber

Eric.Garber@fda.hhs.gov

1 Office of Regulatory Science, Center for Food Safety and Applied Nutrition (CFSAN), Food and Drug Administration, College Park, MD 20740, USA

2 Radix ${ }^{\circledR}$ BioSolutions, Georgetown, TX 78626, USA
} 
TABLE 2: Cross-Reactivity Profile Patterns ${ }^{\text {a }}$

\begin{tabular}{|c|c|c|c|c|c|c|c|c|c|c|c|c|c|c|c|c|c|c|c|c|c|c|c|c|c|c|}
\hline Analyte & $\mathrm{ng} / \mathrm{mL}{ }^{\circ}$ & $\begin{array}{c}\text { Almond- } \\
12^{6}\end{array}$ & $\begin{array}{c}\text { Almond- } \\
13\end{array}$ & $\begin{array}{l}\begin{array}{c}\text { Brazil } \\
\text { nut-14 }\end{array} \\
\end{array}$ & $\begin{array}{l}\text { Brazill } \\
\text { nut-15 }\end{array}$ & $\begin{array}{c}\text { Cashow- } \\
18\end{array}$ & $\begin{array}{c}\text { Cashow- } \\
19\end{array}$ & $\begin{array}{c}\text { Coconut- } \\
20\end{array}$ & $\begin{array}{l}\text { Coconut- } \\
21\end{array}$ & $\begin{array}{c}\text { Crustace } \\
\text { an-22 }\end{array}$ & $\begin{array}{c}\text { Gluten- } \\
27\end{array}$ & 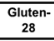 & \begin{tabular}{c|} 
Hazelnut- \\
29
\end{tabular} & $\begin{array}{c}\text { Hazelnut- } \\
30\end{array}$ & $\begin{array}{l}\text { Macada } \\
\text { mia-33 }\end{array}$ & $\begin{array}{c}\text { Macada } \\
\text { mia-34 }\end{array}$ & $\begin{array}{c}\text { Peanut- } \\
37\end{array}$ & $\begin{array}{c}\begin{array}{c}\text { Peanut- } \\
38\end{array} \\
\end{array}$ & $\begin{array}{c}\text { Pine nut- } \\
39\end{array}$ & $\begin{array}{l}\text { Pine nut- } \\
42\end{array}$ & $\begin{array}{c}\text { Pistachi } \\
43\end{array}$ & 10. Pistachio- & Soy-45 & Soy-46 & $\begin{array}{c}\text { Walnut- } \\
47\end{array}$ & $\begin{array}{c}\text { Walnut- } \\
48\end{array}$ \\
\hline \multirow[t]{4}{*}{ Almond } & 10 & 100 & 43 & $<$ & $<$ & 45 & $<$ & $<$ & $<$ & $<$ & $<$ & $6^{d}$ & $<$ & $<$ & 2 & $<$ & $<$ & $<$ & $<$ & $<$ & $<$ & $<$ & $<$ & $<$ & $<$ & $<$ \\
\hline & 25 & 100 & 44 & $\leq$ & $<$ & 47 & $\leq$ & $\leq$ & $\leq$ & $\leq$ & $\leq$ & 6 & $<$ & $<$ & $\leq$ & $\leq$ & $\leq$ & $<$ & $<$ & $\leq$ & $<$ & $\leq$ & $\leq$ & $<$ & $\leq$ & $\leq$ \\
\hline & 50 & 100 & 48 & $<$ & $<$ & 47 & $<$ & $<$ & $<$ & $<$ & $<$ & 5 & 2 & $<$ & 2 & $<$ & $<$ & $<$ & $<$ & $<$ & 2 & $<$ & $<$ & $<$ & $<$ & $<$ \\
\hline & 100 & 100 & 52 & $<$ & $<$ & 47 & $<$ & $<$ & $<$ & < & $<$ & 4 & 3 & 2 & 2 & $<$ & $<$ & $<$ & $<$ & $<$ & 2 & c & $<$ & $<$ & $<$ & $<$ \\
\hline \multirow[t]{4}{*}{ Brazil nut } & 10 & $<$ & $<$ & 100 & 42 & 68 & 21 & $<$ & $<$ & $<$ & $<$ & $<$ & $<$ & 2 & $<$ & $<$ & $<$ & $<$ & $<$ & $<$ & 33 & $<$ & $<$ & $<$ & $<$ & $<$ \\
\hline & 25 & $<$ & $<$ & 100 & 41 & 56 & 15 & $<$ & $<$ & $<$ & $<$ & $<$ & $<$ & 4 & $<$ & $<$ & $<$ & $<$ & $<$ & $<$ & 32 & $<$ & $<$ & $<$ & $<$ & $<$ \\
\hline & 50 & $\therefore$ & $\therefore$ & 100 & 37 & 53 & 15 & $\leqslant$ & $\leq$ & $\leq$ & $\leq$ & $\leq$ & $<$ & 5 & $\therefore$ & $\leq$ & $\leq$ & $<$ & $<$ & $\therefore$ & 30 & $\therefore$ & $\leq$ & $<$ & $<$ & $\leq$ \\
\hline & 100 & $<$ & & 100 & 34 & 50 & 13 & $<$ & $<$ & $<$ & $<$ & $<$ & $<$ & 4 & $<$ & $<$ & $<$ & $<$ & $<$ & $<$ & 27 & $<$ & $<$ & $<$ & $<$ & $<$ \\
\hline \multirow[t]{3}{*}{ Cashew } & 25 & $\leq$ & $<$ & 13 & $<$ & 100 & 48 & $<$ & $<$ & $<$ & $<$ & $<$ & 10 & 2 & $<$ & $<$ & $<$ & $<$ & $<$ & $<$ & 54 & 9 & $<$ & $<$ & 5 & $<$ \\
\hline & $\begin{array}{c}50 \\
100\end{array}$ & $\sum$ & 2 & 14 & $\leq$ & 100 & 50 & $\leq$ & $\leq$ & $\leq$ & $\leq$ & $\leq$ & 10 & 2 & $\leq$ & $\leq$ & $\leq$ & $<$ & $<$ & $<$ & 55 & 10 & $\leq$ & $<$ & 6 & $\therefore$ \\
\hline & 100 & $<$ & $<$ & 21 & $<$ & 100 & 64 & $<$ & < & < & $<$ & $<$ & 16 & 3 & $<$ & $<$ & $<$ & $<$ & $<$ & $<$ & 66 & 16 & $<$ & $<$ & 8 & 2 \\
\hline \multirow[t]{3}{*}{ Coconut } & 25 & $<$ & $<$ & $<$ & $<$ & $<$ & $<$ & 100 & 4 & $<$ & $<$ & $<$ & $<$ & $<$ & 13 & $<$ & $<$ & $<$ & $<$ & $<$ & $<$ & $<$ & $<$ & $<$ & $<$ & $<$ \\
\hline & $\begin{array}{r}50 \\
100\end{array}$ & $\xi$ & ¿ & $<$ & $<$ & $<$ & $<$ & 100 & 5 & $<$ & $<$ & $<$ & $<$ & $<$ & 5 & $<$ & $<$ & $<$ & $<$ & $<$ & $<$ & $<$ & $<$ & $<$ & $<$ & $<$ \\
\hline & 100 & c & c & $<$ & $<$ & $<$ & $<$ & 100 & 4 & < & $<$ & $<$ & $<$ & $<$ & 5 & $<$ & $<$ & $<$ & $<$ & $<$ & $<$ & $<$ & $<$ & $<$ & $<$ & $<$ \\
\hline \multirow[t]{4}{*}{ Crustacean } & 10 & 2 & $<$ & $<$ & $<$ & $<$ & $<$ & 2 & $<$ & 100 & $<$ & $<$ & 2 & $<$ & $<$ & $<$ & $<$ & $<$ & $<$ & $<$ & $<$ & $<$ & $<$ & $<$ & $<$ & $<$ \\
\hline & 25 & 2 & $<$ & $<$ & $<$ & $<$ & $<$ & 2 & $<$ & 100 & $<$ & $<$ & 2 & $<$ & $<$ & $<$ & $<$ & 2 & $<$ & $<$ & 2 & $<$ & $<$ & $<$ & $<$ & $<$ \\
\hline & 50 & 3 & $<$ & 2 & $<$ & $<$ & 2 & 3 & 2 & 100 & 2 & 2 & 2 & $<$ & $<$ & $<$ & 2 & 2 & $<$ & $<$ & 2 & 2 & 2 & 2 & 2 & $<$ \\
\hline & 100 & 2 & $<$ & $<$ & $<$ & $<$ & $<$ & 2 & $<$ & 100 & $<$ & $<$ & 2 & $<$ & $<$ & $<$ & 2 & 2 & $<$ & $<$ & 2 & $<$ & $<$ & $<$ & $<$ & $<$ \\
\hline \multirow[t]{4}{*}{ Gluten } & 10 & $<$ & $<$ & $<$ & $<$ & $<$ & $<$ & $<$ & $<$ & $<$ & 15 & 100 & $<$ & $<$ & $<$ & $<$ & $<$ & $<$ & $<$ & $<$ & $<$ & $<$ & $<$ & $<$ & $<$ & $<$ \\
\hline & 25 & $<$ & $<$ & $<$ & $<$ & $<$ & $<$ & $<$ & $<$ & $<$ & 16 & 100 & $<$ & $<$ & $<$ & $<$ & $<$ & $<$ & $<$ & $<$ & $<$ & $<$ & $<$ & $<$ & $<$ & $<$ \\
\hline & 50 & $<$ & < & $<$ & $<$ & $<$ & $<$ & $<$ & $<$ & $<$ & 16 & 100 & $<$ & $<$ & $<$ & $<$ & $<$ & $<$ & $<$ & $<$ & $<$ & e & $<$ & $<$ & $<$ & $<$ \\
\hline & 100 & $<$ & $<$ & $<$ & $<$ & $<$ & $<$ & $<$ & $<$ & $<$ & 18 & 100 & $<$ & $<$ & $<$ & $<$ & $<$ & $<$ & $<$ & $<$ & $<$ & $<$ & $<$ & $<$ & $<$ & $<$ \\
\hline \multirow[t]{4}{*}{ Hazelnut } & 10 & $<$ & $<$ & $<$ & $<$ & 22 & 5 & $<$ & $<$ & $<$ & $<$ & $<$ & 100 & 18 & 2 & s & $<$ & $<$ & $<$ & $<$ & 7 & s & $<$ & $<$ & $<$ & $<$ \\
\hline & 25 & $<$ & $<$ & $<$ & $<$ & 24 & 7 & $<$ & $<$ & $<$ & $<$ & $<$ & 100 & 19 & 3 & $<$ & $<$ & $<$ & $<$ & $<$ & 9 & $<$ & $<$ & $<$ & $<$ & < \\
\hline & 50 & $<$ & $<$ & $<$ & $<$ & 26 & 7 & $<$ & $<$ & $<$ & $<$ & $<$ & 100 & 20 & 4 & $<$ & $<$ & $<$ & $<$ & $<$ & 9 & $<$ & $<$ & $<$ & $<$ & $<$ \\
\hline & 100 & $<$ & $<$ & 2 & $<$ & 30 & 9 & $<$ & $<$ & $<$ & $<$ & $<$ & 100 & 23 & 5 & $<$ & $<$ & $<$ & $<$ & $<$ & 10 & $<$ & $<$ & $<$ & $<$ & 2 \\
\hline \multirow[t]{4}{*}{ Macadamia } & 10 & e & e & $<$ & $<$ & $<$ & e & e & e & e & $<$ & $<$ & $<$ & e & 100 & 15 & < & $<$ & $<$ & c & e & e & $<$ & $<$ & $<$ & e \\
\hline & 25 & $<$ & $<$ & $<$ & $<$ & $<$ & $<$ & $<$ & $<$ & $<$ & $<$ & $<$ & $<$ & $<$ & 100 & 12 & $<$ & $<$ & $<$ & $<$ & $<$ & $<$ & $<$ & $<$ & $<$ & $<$ \\
\hline & 50 & $<$ & $<$ & $<$ & $<$ & $<$ & $<$ & $<$ & $<$ & $<$ & $<$ & $<$ & $<$ & $<$ & 100 & 10 & $<$ & $<$ & $<$ & $<$ & $<$ & $<$ & $<$ & $<$ & $<$ & $<$ \\
\hline & 100 & $<$ & $<$ & $<$ & $<$ & $<$ & $<$ & $<$ & $<$ & $<$ & $<$ & $<$ & $<$ & $<$ & 100 & 11 & $<$ & $<$ & $<$ & $<$ & $<$ & $<$ & $<$ & $<$ & $<$ & $<$ \\
\hline \multirow[t]{4}{*}{ Peanut } & 10 & 2 & $<$ & 2 & $<$ & 14 & 3 & 2 & $<$ & $<$ & $<$ & $<$ & $<$ & $<$ & $<$ & $<$ & 69 & 100 & $<$ & $<$ & 2 & $<$ & 4 & 4 & $<$ & $<$ \\
\hline & 25 & 4 & $<$ & 3 & $<$ & $\begin{array}{l}18 \\
18\end{array}$ & 5 & 4 & $<$ & $<$ & $<$ & $<$ & $<$ & $<$ & 3 & $<$ & 70 & 100 & $<$ & $<$ & 4 & $<$ & 6 & 6 & 2 & $<$ \\
\hline & 50 & 6 & $<$ & 4 & $<$ & 22 & 6 & 6 & $<$ & $<$ & $<$ & $<$ & 2 & 2 & 4 & $<$ & 71 & 100 & 2 & $<$ & 5 & 2 & 8 & 7 & 4 & $<$ \\
\hline & 100 & 10 & 2 & 7 & $<$ & 27 & 8 & 10 & $<$ & 2 & 2 & 2 & 5 & 3 & 7 & $<$ & 73 & 100 & 4 & $<$ & 8 & 3 & 11 & 10 & 7 & 3 \\
\hline \multirow[t]{4}{*}{ Pine nut } & 10 & $<$ & $<$ & $<$ & $<$ & $<$ & $<$ & $<$ & $<$ & $<$ & $<$ & $<$ & $<$ & $<$ & $<$ & $<$ & $<$ & $<$ & 100 & 2 & $<$ & $<$ & $<$ & $<$ & $<$ & $<$ \\
\hline & 25 & < & $=$ & $<$ & $<$ & $<$ & $<$ & $<$ & $<$ & $<$ & $<$ & $<$ & $<$ & $<$ & $<$ & $=$ & $<$ & $<$ & 100 & 5 & $=$ & $=$ & $<$ & $<$ & $<$ & $<$ \\
\hline & 50 & $\therefore$ & $<$ & $<$ & $<$ & $<$ & $<$ & $\therefore$ & $<$ & $\therefore$ & $<$ & $<$ & $<$ & $<$ & $\therefore$ & 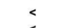 & $<$ & $<$ & 100 & 7 & $\therefore$ & $\therefore$ & $<$ & $<$ & $<$ & 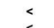 \\
\hline & 100 & $<$ & $<$ & $<$ & $<$ & $<$ & $<$ & $<$ & $<$ & $<$ & $<$ & $<$ & $<$ & $<$ & $<$ & $<$ & $<$ & $<$ & 100 & 9 & $<$ & $<$ & $<$ & $<$ & $<$ & $<$ \\
\hline \multirow[t]{4}{*}{ Pistachio } & 10 & $<$ & $<$ & 18 & $<$ & 87 & 24 & $<$ & $<$ & $<$ & $<$ & $<$ & 4 & $<$ & $<$ & $<$ & $<$ & $<$ & $<$ & $<$ & 100 & 38 & $<$ & $<$ & $<$ & $<$ \\
\hline & 25 & c & $c$ & 19 & $<$ & 95 & 26 & $c$ & $<$ & $<$ & $<$ & $<$ & 5 & $<$ & $<$ & $<$ & $<$ & $<$ & $c$ & $c$ & 100 & 39 & $<$ & $<$ & $<$ & $<$ \\
\hline & 50 & $<$ & $<$ & 20 & $<$ & 97 & 29 & $<$ & $<$ & $<$ & $<$ & $<$ & 7 & $<$ & $<$ & $<$ & $<$ & $<$ & $<$ & $<$ & 100 & 42 & $<$ & $<$ & 2 & $<$ \\
\hline & 100 & $<$ & $<$ & 22 & $<$ & 92 & 30 & $<$ & $<$ & $<$ & $<$ & $<$ & 8 & $<$ & $<$ & $<$ & $<$ & $<$ & $<$ & $<$ & 100 & 45 & $<$ & $<$ & 2 & $<$ \\
\hline Soy & 10 & $<$ & $=$ & 5 & $<$ & 22 & 3 & $<$ & c & $<$ & $<$ & $<$ & 2 & $<$ & $<$ & $<$ & $<$ & 3 & $<$ & c & 9 & c & 100 & 69 & $<$ & $<$ \\
\hline & 25 & 2 & e & 7 & $<$ & 27 & 5 & $<$ & $<$ & $<$ & $<$ & $<$ & 3 & $<$ & $<$ & $<$ & $<$ & 4 & $<$ & e & 12 & < & 100 & 67 & $<$ & 2 \\
\hline & 50 & 3 & $<$ & 9 & $<$ & 32 & 6 & 2 & $<$ & $<$ & $<$ & 2 & 5 & $<$ & $\leq$ & $<$ & $<$ & 5 & $<$ & $<$ & 16 & 2 & 100 & 70 & 2 & 2 \\
\hline & 100 & 5 & $<$ & 14 & $<$ & 43 & 9 & 3 & $<$ & $<$ & 2 & 4 & 8 & $<$ & 2 & $<$ & 2 & 8 & $<$ & $<$ & 25 & 2 & 100 & 74 & 3 & 4 \\
\hline Walnut & 10 & $<$ & $<$ & 3 & $<$ & 7 & $<$ & $<$ & $<$ & < & $<$ & $<$ & 1 & $<$ & c & $<$ & $<$ & $<$ & $<$ & $<$ & 3 & $<$ & $<$ & $<$ & 100 & 78 \\
\hline & 25 & $<$ & $=$ & 5 & $<$ & 11 & $<$ & $=$ & $=$ & $=$ & $<$ & $<$ & 5 & $<$ & $=$ & $<$ & $<$ & $<$ & $<$ & $=$ & 4 & $=$ & $<$ & $<$ & 100 & 83 \\
\hline & 50 & $\leqslant$ & $\leq$ & 4 & $\Sigma$ & 8 & 2 & $\Sigma$ & $\leqslant$ & $\leqslant$ & 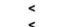 & $\Sigma$ & 5 & $\leqslant$ & $\Sigma$ & $\leq$ & $\leqslant$ & $\leqslant$ & $\leq$ & $\Sigma$ & 3 & $\leq$ & 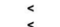 & $\leq$ & 100 & 84 \\
\hline
\end{tabular}

- Responses of non-denatured analyses tabulated as average response minus background, normalized to the most intense bead set for the analyte, set to 100 (i.e., Almond-12, Cashew-18, Coconut-20, Crustacean-22, Gluten-28, Hazelnut-29, Macadamia-33, Peanut-38, Pine nut-39, Pistachio-

'Suspected artifact of Gluten-28-these responses were within the noise and below the dynamic range of gluten-28. Though when normailized relative to Almond appear as indicated.

- Suspected noise error in data generation. 